Portland State University

PDXScholar

\title{
Changes in Personality Traits and Identification in Adolescent Female State School Residents, as a Function of Length of Residence
}

Mary L. Thompson

Portland State University

Follow this and additional works at: https://pdxscholar.library.pdx.edu/open_access_etds

Part of the Child Psychology Commons, Personality and Social Contexts Commons, and the Social Work Commons

Let us know how access to this document benefits you.

\section{Recommended Citation}

Thompson, Mary L., "Changes in Personality Traits and Identification in Adolescent Female State School Residents, as a Function of Length of Residence" (1973). Dissertations and Theses. Paper 1756. https://doi.org/10.15760/etd.1755

This Thesis is brought to you for free and open access. It has been accepted for inclusion in Dissertations and Theses by an authorized administrator of PDXScholar. Please contact us if we can make this document more accessible: pdxscholar@pdx.edu. 
CHANGES IN PERSONALITY TRAITS AND IDENTIFICATION

IN ADOLESCENT FEMALE STATE SCHOOL RESIDENTS,

AS A FUNCTION DF LENGTH OF RESIDENCE

by

MARY L. THOMPSON

A report submitted in partial fulfillment of the requirements for the degree of

MASTER OF

SOCIAL WORK

Portland State University

1973 


\section{ACKNOWLEDGEMENTS}

My fullest thanks goes to the girls of Maple Lane School. Their willingness to participate, perseverance and expenditure of time made this study possible.

I am very grateful to the staff of Maple Lane and especially to J. Richard Barrett and Richard L. Leitch. They provided the opportunity to make this study, opened their program to inspection and extended theix assistance throughout the project.

For his patience, faith and desire that I learn, my deepest appreciation is to Eric Guy Thompson, my husband and consultant.

$$
\text { M.L.T. }
$$


Page

List of Tables • • • • • • • • • • • •

List of figures ... . . . . . . . . . . viii

Introduction

Method

Results

Conclusions

Selected Bibliography

Appendices

1 California Psychological Inventory Scales: Brief Definition and Purpose. . - •

2 The Jesness Inventory: Brief Definition of the Scales 


\section{LIST OF TABLES}

Table

Page

1 Raw Score Results on the CPI over Six Months............ 7

2 Raw Score Results on the Jesness Inventory over Six Months ........... 8

3 T-Score Means on the Jesness Inventory..$\quad B$

4 Initial Comparison of Sample and CPI Norm Group . . . . . . . . . . 9

5 Six-Month Comparison of Sample and CPI Norm Group............ 10

6 Analysis of Variance for the Dominance Scale ..............

7 Analysis of Variance for the Capacity for Status Scale...........

8 Analysis of Variance for the Sociability Scale ..............

9 Analysis of Variance for the Social Presence Scale...........

10 Analysis of Variance for the SelfAcceptance Scale .......... 12

11 Analysis of Variance for the Well-being Scale .............. .

12 Analysis of Variance for the Responsibility Scale................

13 Analysis of Variance for the Socialization Scale..............

14 Analysis of Variance for the Self-Control Scale............... 14

15 Analysis of Variance for the Tolerance Scale............ 14 
Table

Page

16 Analysis of Variance for the Good Impressian Scale... . . . . .

17 Analysis of Variance for the Communality Scale... . . . . . . . . . ..

18 Analysis of Variance for the Achievement via Conformity Scale... . . . .

19 Analysis of Variance for the Achievement via Independence Scale... . . . .

20 Analysis of Variance for the Intellectual Efficiency Scale. . . . . . . . .

21 Analysis of Variance for the Psychologicalmindedness Scale . . . . . . . .

22 Analysis of Variance for the Flexibility Scale.................

23 Analysis of Variance for the Femininity

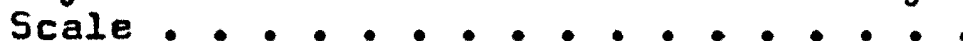

24 Analysis of Variance for the Social

Maladjustment Scale (Weighted Items). .

25 Analysis of Variance for the Social

Maladjustment Scale... . . . . .

26 Analysis of Variance for the Value Orientation Scale . . . . . . . . .

27 Analysis of Variance for the Immaturity Scale .... . . . . . . . . .

28 Analysis of Variance for the Autism Scale....................

29 Analysis of Variance for the Alienation Scale . . . . . . . . . . . .

30 Analysis of Variance for the Manifest Aggression Scale...........

31 Analysis of Variance for the Withdrawal Scale................

32 Analysis of Variance for the Social Anxiety Scale... . . . . . . . 
33 Analysis of Variance for the Repression Scale... . . . . . . . . . .

34 Analysis of Variance for the Denial

$$
\text { Scele................. }
$$

35 Analysis of Variance for the Asocial Index . . . . . . . . . . . .

36 Variance Ratios on the CPI over Three Administrations . . . . . . . . .

37 Variance Ratios on the Jesness Inventory . . . . . . . . . - 


\section{LIST OF FIGURES}

Figure

Page

1 High, Middle and Low Scorers on the Asocial Index over Six Months...

2 Changes in Delinquency Proneness in High Scorers... . . . . . . .

3 Changes in Delinquency Proneness in Middle Scorers . . . . . . . . .

4 Changes in Delinquency Proneness in Low Scorers............. 
The purpose of this study was to assess the impact of a significant exposure (six months) to a State School environment on selected personality traits and identity factors of adolescent girls. A second abjective was to ascertain whether greater change takes place early in the period of residency (three months) or in a later stage. A third aim was to determine whether there is a significant difference in the degree of change between girls showing fewer pathological signs and healthier identity than those who show a greater number of such signs and a stronger delinquent identification.

The repeated measurements model, using personality inventories, has been used extensively in studies of the effectiveness of treatment. Frequently, the findings show little or no impact from the treatment intervention. Similar results are reported in the field of juvenile delinquency, covering a range from psychotherapy (Guttman, 1961 ) to differences in size of living groups (Jesness, 1965). These studies found no significant mean differences in the amount of change between experimental and control groups; but did not take into account the possibility of significant change in the variability of outcome.

In re-analyzing the data of psychotherapy outcome studies, Bergin $(1963,1966)$ reports the phenomenon of 
experimental groups attaining a much wider dispersion of scores than control groups, even though the mean change in both groups is quite similar. In other words, experimental subjects were typically dispersed from "marked improvement" to "marked deterioration"; while in the control subjects, varying amounts of change clustered around the mean.

Bergin (1970) in defending his findings and concept of a "deterioration effect" comments upon the potency of psychotherapy and its potential to have both harmful and beneficial effects. It is speculated that institutional treatment has an even greater impact than psychotherapy due to the more pervasive change in the subject's life situation.

The implications of Bergin's research would clearly suggest that young people will become better, or worse, in adjustment as the result of institutional experience. One way of attempting to deal with the problem is to institute differential treatment modalities for juvenile offenders on the basis of predicted differential reactions to treatment (Warren, 1966; Jesness, 1971; and Campos, 1967). For Maple Lane School, the initial task is to determine whether or not its program is helpful to some, while detrimental to others; and to whom, under what circumstances. 
METHOD

Setting

This study took place at Maple Lane School, an institution for girls committed to the Department of Social and Health Services, State of Washington. The School has nine treatment cottages and a bed capacity for $146 \mathrm{girls}$. It is one of several state institutions for youth and has been used primarily as a resource "for the older girls and those who are more seriously delinquent and sophisticated" (Office of Research, Division of Institutions, 1970).

For the fiscal year 1972, the average daily population was 142.8 , excluding 23.1 girls on AWOL status. The average length of stay (calendar year) of girls being released was 14 months, 7.3 days: a figure which includes the length of time a girl is away from campus on unauthorized leave and furloughs. There were 119 new admissions to the School, 36 per cent of whom were girls previously committed to the state' system.*

\section{Subjects}

Twenty-eight girls consecutively admitted to Maple Lane School for the first time during July, August and September 1972, were asked to participate in the study. Participation was voluntary; two students declined initially and two dropped out later. Their reasons were

* The data was supplied by the Department of Social Service, Maple Lane School. 
twofold: "It takes too long." and "I just don't want to." Three girls were functionally illiterate and were unable to read the questions. An eighth girl was dropped from the sample as she was released before the completion of the project. Aside from inability to read, no other selecting factor could be identified in those girls who did not participate.

The sample consists then of twenty girls ranging in age from thirteen to eighteen at the time of admission. Each had been adjudicated "dependent/incorrigible" and had spent at least six weeks in the State's Diagnostic Center. Their difficulties ranged from inability to adjust in foster care to conflicts with the law in what would be felony offenses for adults. Three had been paroled from other state schools and then returned from the community.

Measurements

The two personality inventories chosen for this study have been used in conjunction with other indices in several California studies of juveniles. They both have been reported to distinguish delinquents from nondelinquents throughout the United 5 tates, as well as in England (Rose, 1967).

The Jesness Inventory is a structured personalityattitude test of ten scales devised expressly for the purposes of measuring dimensions related to delinquency proneness, the classification of delinquents into types 
and evaluation of change. The Asocial Index numerically approximates Jesness' concept that unmet needs, minus inhibitory tendencies, equates with a psychological disposition toward delinquency (Jesness, 1963).

A well established personality inventory was needed to use as a recognized yardstick against which change could be measured. The absence of pathology in an individual does not necessarily insure that he is welladjusted. Gough (1957) states the California Psychological Inventory (CPI) is primarily designed for use with non-psychiatrically disturbed subjects. The focus of the scales is on those characteristics of personality that are important for social living and social interaction. His description and the choice of the CPI for this study are supported by the findings of Haan (1965), whose research indicated that the CPI is a more efficient measure of coping mechanisms (good ego functioning) than the MMPI: the latter measuring the poorer functioning of defense mechanisms.

The limitations of this study, in both time and in the size of the sample, precluded making the full use of potential of the CPI. All eighteen of the scales were used; however, Gough's more complex Social Maturity Index was not computed (Gough, 1971), nor was a factor analysis attempted (Megargee, 1972).

\section{Procedure}

The original intent was to administer the invento- 
ries to each girl within one week of her arrival at Maple Lane. With all but three girls, the tests were completed earlier and within two days of admission. Each girl was told that the purpose of the study was to compare a group of Maple Lane girls with other groups of girls who had taken the tests; and each was given the standard set of instructions from the respective manuals.

The inventories were given a second time to each girl after she had been in the institution for three months (thirteen weeks, two days). The final administration of the two tests was completed for each girl after she had been at Maple Lane for a period of six months (twenty-six weeks). Due to reasons of illness and furloughs, four girls were tested in the twentyseventh and twenty-eighth week.

Early in the study, it became apparent that a number of subjects were scoring so low on the Communality (Cm) scale, a measure of validity, that their responses were questionable. This seemed to be due to some of the girls "faking bad". It has been shown that there is a positive relationship between the ability to "fake good" on the CPI and the actual life adjustment of the subject (Canter, 1963). It was decided to retain those tests where the subjects answered in such a way as to emphasize their personal problems. To discard them would have distorted the results to make the girls appear better adjusted than they were. 


\section{RESULTS}

Tables 1 and 2 show the means and standard deviations on the CPI and Jesness Inventory for the three administrations. Table 3 shows the mean T-scores on the Jesness Inventory for those scales for which female norms are available.

Table 1

Raw Score Results on the CPI over Six Months

\begin{tabular}{|c|c|c|c|c|c|c|}
\hline \multirow{2}{*}{$\frac{\text { Scale }}{\text { Do }}$} & \multicolumn{2}{|c|}{$\begin{array}{l}\text { Initial } \\
\text { M. } \quad 5 . D . \\
\end{array}$} & \multicolumn{2}{|c|}{ 3-Month } & \multicolumn{2}{|c|}{ 6-Month } \\
\hline & 24.3 & 6.5 & 23.4 & 6.5 & 25.2 & 6.6 \\
\hline Cs & 13.6 & 3.8 & 14.2 & 4.5 & 14.2 & 4.7 \\
\hline $5 y$ & 20.0 & 5.0 & 20.2 & 6.1 & 21.4 & 5.5 \\
\hline$S p$ & 32.6 & 3.7 & 31.7 & 5.7 & 33.3 & 5.1 \\
\hline $5 a$ & 19.9 & 2.9 & 19.6 & 3.2 & 20.6 & 3.2 \\
\hline Wb & 26.0 & 8.0 & 27.8 & 7.9 & 28.7 & 7.2 \\
\hline $\operatorname{Re}$ & 20.0 & 5.3 & 21.5 & 6.1 & 22.2 & 5.5 \\
\hline So & 24.3 & 5.9 & 26.8 & 4.8 & 25.7 & 6.2 \\
\hline Se & 20.2 & 8.0 & 21.2 & 7.0 & 20.9 & 8.3 \\
\hline To & 13.5 & 5.3 & 15.4 & 5.9 & 15.4 & 5.9 \\
\hline Gi & 13.0 & 6.0 & 12.3 & 6.5 & 12.0 & 7.1 \\
\hline $\mathrm{Cm}$ & 22.1 & 5.6 & 23.2 & 5.0 & 23.7 & 4.8 \\
\hline Ac & 19.4 & 4.6 & 20.2 & 4.5 & 19.9 & 6.7 \\
\hline$A i$ & 14.1 & 4.0 & 15.5 & 4.4 & 15.2 & 4.1 \\
\hline Ie & 28.6 & 6.0 & 27.9 & 6.3 & 30.9 & 7.7 \\
\hline Py & 8.7 & 2.7 & 9.5 & 3.0 & 9.5 & 3.9 \\
\hline$F x$ & 8.9 & 3.5 & 10.1 & 3.6 & 9.3 & 4.3 \\
\hline Fe & 19.8 & 3.1 & 20.9 & 3.4 & 20.3 & 2.3 \\
\hline
\end{tabular}


Table 2

Raw Score Results on the Jesness Inventory over Six Months

\begin{tabular}{|c|c|c|c|c|c|c|}
\hline Scale & \multicolumn{2}{|c|}{ Initial } & \multicolumn{2}{|c|}{ 3-Month } & \multicolumn{2}{|c|}{ 6-Month } \\
\hline $5 M x$ & 13.6 & 5.5 & 11.5 & 5.4 & 11.7 & 6.6 \\
\hline SM & 26.4 & 5.9 & 24.9 & 6.2 & 24.6 & 8.1 \\
\hline Vo & 14.6 & 8.0 & 14.3 & 7.9 & 13.5 & 8.7 \\
\hline$I \mathrm{~mm}$ & 12.7 & 4.0 & 12.5 & 4.8 & 12.9 & 3.8 \\
\hline $\mathrm{Au}$ & 9.9 & 4.2 & 8.3 & 4.0 & B. 6 & 3.3 \\
\hline Al & 7.1 & 4.0 & 6.8 & 5.3 & 6.9 & 4.5 \\
\hline MA & 15.6 & 6.9 & 16.5 & 5.7 & 14.2 & 6.8 \\
\hline Wd & 13.3 & 3.3 & 12.9 & 2.9 & 12.8 & 3.7 \\
\hline$S A$ & 14.6 & 4.5 & 15.6 & 3.0 & 14.8 & 5.4 \\
\hline Rep & 3.5 & 2.3 & 2.4 & 2.4 & 2.6 & 1.9 \\
\hline Den & 10.1 & 4.7 & 10.7 & 4.3 & 10.4 & 5.1 \\
\hline$A / I$ & 25.1 & 3.2 & 23.8 & 4.2 & 23.9 & 5.6 \\
\hline
\end{tabular}

Table 3

T-Score Means on the Jesness Inventory

\begin{tabular}{lccc}
\hline Scale & Initial & 3-Month & 6-Month \\
\hline SM & 62.4 & 60.6 & 60.2 \\
Vo & 52.3 & 52.0 & 48.2 \\
Imm & 51.7 & 50.3 & 52.6 \\
Au & 59.2 & 55.4 & 56.7 \\
Al & 53.8 & 52.0 & 52.6 \\
MA & 52.7 & 50.9 & 50.0 \\
Wd & 53.2 & 52.0 & 51.7 \\
SA & 48.2 & 51.0 & 48.9 \\
Rep & 49.9 & 43.7 & 45.8 \\
Den & 45.9 & 47.0 & 47.1 \\
\hline
\end{tabular}


Norms for high school girls (Gough, 1957) were used instead of general norms because the latter include the relatively higher scores of college and professional women. This decision is further supported by the fact that Maple Lane girls are drawn from a high school-age population.

Table 4 shows the means and standard deviations of the norm group plus the mean differences between the sample and the norms at the time of admission to Maple Lane School.

\section{Table 4}

Initial Comparison of Sample and CPI Norm Group

\begin{tabular}{|c|c|c|c|c|c|}
\hline \multicolumn{3}{|c|}{$\begin{array}{l}\text { High School students } \\
(N=4,056)\end{array}$} & \multicolumn{2}{|c|}{$\begin{array}{l}\text { Sample } \\
(N=20)\end{array}$} & \\
\hline Scale & M. & 5.0. & M.diff & $t$ & \\
\hline $\begin{array}{l}\text { Do } \\
\text { Cs } \\
\text { Sy } \\
\text { Sp } \\
\text { Sa } \\
\text { Wb }\end{array}$ & $\begin{array}{l}23.7 \\
16.0 \\
21.4 \\
31.1 \\
18.9 \\
34.6\end{array}$ & $\begin{array}{l}6.1 \\
4.9 \\
5.7 \\
5.8 \\
4.4 \\
5.7\end{array}$ & $\begin{array}{l}.6+ \\
2.4 \\
1.4 \\
1.5+ \\
1.0+ \\
8.6\end{array}$ & $\begin{array}{l}.411 \\
2.791 \\
1.260 \\
1.798 \\
1.543 \\
4.802\end{array}$ & $\begin{array}{l}* * \\
* \\
* *\end{array}$ \\
\hline $\begin{array}{l}\text { Re } \\
\text { So } \\
\text { Sc } \\
\text { To } \\
\text { Gi } \\
\text { Cm }\end{array}$ & $\begin{array}{l}30.0 \\
39.4 \\
27.6 \\
18.7 \\
15.7 \\
26.1\end{array}$ & $\begin{array}{l}5.2 \\
5.6 \\
8.5 \\
5.5 \\
6.2 \\
1.9\end{array}$ & $\begin{array}{r}10.0 \\
15.1 \\
7.4 \\
5.2 \\
2.7 \\
4.0\end{array}$ & $\begin{array}{r}8.382 \\
11.370 \\
4.131 \\
4.388 \\
2.010 \\
3.208\end{array}$ & $\begin{array}{l}\text { ** } \\
* * \\
* * \\
* * \\
* \\
* *\end{array}$ \\
\hline $\begin{array}{l}A c \\
A i \\
I e \\
P y \\
F x \\
F e\end{array}$ & $\begin{array}{r}24.1 \\
15.5 \\
34.4 \\
8.7 \\
8.9 \\
24.1\end{array}$ & $\begin{array}{l}5.3 \\
4.2 \\
6.5 \\
2.6 \\
3.2 \\
3.5\end{array}$ & $\begin{array}{l}4.7 \\
1.4 \\
5.8 \\
0.0 \\
0.0 \\
4.3\end{array}$ & $\begin{array}{c}4.568 \\
1.547 \\
4.335 \\
--- \\
6.205\end{array}$ & $\begin{array}{l}* * \\
* * \\
* *\end{array}$ \\
\hline $\begin{array}{l}+ \text { samp } \\
* p< \\
{ }^{*} p<\end{array}$ & scc & , & $m g r$ & & \\
\hline
\end{tabular}


The subjects did not differ significantly from normal high school girls on six CPI scales. Sample means were significantly lower (indicating a greater degree of maladjustment) on eleven scales, and were significantly higher (indicating greater functioning) on one scale.

- Table 5 shows the same comparisons at the sixmonth stage. Maple Lane students continued to differ significantly from the norm group on the same twelve scales, in addition to the Self-acceptance (Sa) scale. Differences between sample and norm means decrease, indicating improved adjustment on all but one scale.

Table 5

Six-Month Comparison of Sample and CPI Norm Group

\begin{tabular}{|c|c|c|c|c|c|}
\hline \multicolumn{3}{|c|}{$\begin{array}{c}\text { High School Students } \\
(N=4,056)\end{array}$} & \multicolumn{3}{|c|}{$\begin{array}{l}\text { Maple Lane Students } \\
\qquad(N=20)\end{array}$} \\
\hline Scale & M. & S.D. & 3-Month M.diff & 6-Month & M. diff \\
\hline $\begin{array}{l}\text { Do } \\
\text { Cs } \\
\text { Sy } \\
\text { Sp } \\
\text { Sa } \\
\text { Wb }\end{array}$ & $\begin{array}{l}23.7 \\
16.0 \\
21.4 \\
31.1 \\
18.9 \\
34.6\end{array}$ & $\begin{array}{l}6.1 \\
4.9 \\
5.7 \\
5.8 \\
4.4 \\
5.7\end{array}$ & $\begin{array}{r}+.6 \\
2.4 \\
1.4 \\
+1.5 \\
+1.0 \\
8.6\end{array}$ & $\begin{array}{r}+1.5 \\
1.8 \\
0.0 \\
+2.2 \\
+1.7 \\
5.9\end{array}$ & $\begin{array}{l}* \\
* \\
* \\
*\end{array}$ \\
\hline $\begin{array}{l}\text { Re } \\
\text { So } \\
\text { Sc } \\
\text { To } \\
\text { Gi } \\
C_{m}\end{array}$ & $\begin{array}{l}30.0 \\
39.4 \\
27.6 \\
18.7 \\
15.7 \\
26.1\end{array}$ & $\begin{array}{l}5.2 \\
5.6 \\
8.5 \\
5.5 \\
6.2 \\
1.9\end{array}$ & $\begin{array}{r}10.0 \\
15.1 \\
7.4 \\
5.2 \\
2.7 \\
4.0\end{array}$ & $\begin{array}{r}7.8 \\
13.7 \\
6.7 \\
3.3 \\
3.7 \\
2.4\end{array}$ & $\begin{array}{l}* * \\
* * \\
* * \\
* \\
* \\
*\end{array}$ \\
\hline $\begin{array}{l}A c \\
A i \\
I e \\
P y \\
F x \\
F e\end{array}$ & $\begin{array}{r}24.1 \\
15.5 \\
34.4 \\
8.7 \\
8.9 \\
24.1\end{array}$ & $\begin{array}{l}5.3 \\
4.2 \\
6.5 \\
2.6 \\
3.2 \\
3.5\end{array}$ & $\begin{array}{l}4.7 \\
1.4 \\
5.8 \\
0.0 \\
0.0 \\
4.3\end{array}$ & $\begin{array}{r}4.2 \\
.3 \\
3.5 \\
+\quad .8 \\
+.4 \\
3.8\end{array}$ & $\left\{\begin{array}{l}* * \\
* \\
* *\end{array}\right.$ \\
\hline $\begin{array}{l}+ \text { san } \\
t \text { tes }\end{array}$ & 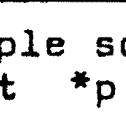 & 05 & $\begin{array}{l}\text { er than norm } \\
\rho<.01 .\end{array}$ & group. & \\
\hline
\end{tabular}


Tables 6 through 23 show analyses for trends on the CPI. In no case did trials variability achieve a level of significance; thereby indicating no mean difference occurred due to the effect of time in the institution. Subject variability is significant on all of the eighteen scales, as expected, indicating the subjects are not homogeneous with respect to personality traits.

Table 6

Analysis of Variance for the Dominance Scale

\begin{tabular}{|c|c|c|c|c|}
\hline $\begin{array}{l}\text { Source of } \\
\text { Variation }\end{array}$ & Sum of Squares & $d f$ & Mean Square & $\boldsymbol{F}$ \\
\hline Trials & 34.0 & 2 & 17.0 & .890 \\
\hline Subjects & 1732.1 & 19 & 91.2 & $4.775 * *$ \\
\hline S's X trials & 724.9 & 38 & 19.1 & \\
\hline Total & 2491.0 & 59 & & \\
\hline
\end{tabular}

Table 7

Analysis of Variance for the Capacity for Status Scale

\begin{tabular}{lrrrr}
\hline $\begin{array}{l}\text { Source of } \\
\text { Variation }\end{array}$ & Sum of Squares & df & Mean Square & $F$ \\
\hline Trials & 4.4 & 2 & 2.2 & .301 \\
Subjects & 801.5 & 19 & 42.2 & $5.780^{*}$ \\
S's X trials & $\frac{278.1}{1084.0}$ & $\frac{38}{59}$ & 7.3 & \\
\multicolumn{1}{l}{ Total } & 10 & & \\
\hline
\end{tabular}

* $p<.05$.

** $\mathrm{p}<.01$. 
Table 8

Analysis of Variance for the Sociability Scale

\begin{tabular}{|c|c|c|c|c|}
\hline $\begin{array}{l}\text { Source of } \\
\text { Variation }\end{array}$ & Sum of Squares & $d f$ & Mean Square & $\mathbf{F}$ \\
\hline Trials & 12.8 & 2 & 6.4 & .598 \\
\hline Subjects & 1301.5 & 19 & 68.5 & $6.401 * *$ \\
\hline s's $X$ trials & 406.3 & 38 & 10.7 & $\cdot$ \\
\hline Total & 1720.6 & 59 & & \\
\hline
\end{tabular}

Table 9 Analysis of Variance for the Social
Presence Scale

\begin{tabular}{lrrrr}
\hline $\begin{array}{l}\text { Source of } \\
\text { Variation }\end{array}$ & Sum of Squares & of & Mean Square & $F$ \\
\hline Trials & 25.5 & 2 & 12.7 & 4.379 \\
Subjects & 1265.5 & 19 & 66.0 & $22.750^{*}$ \\
Sis X trials & $\frac{108.7}{1399.7}$ & $\frac{38}{59}$ & 2.9 & \\
Total & 139 & & \\
\hline
\end{tabular}

Table 10

Analysis of Variance for the Self-acceptance Scale

\begin{tabular}{lccccc}
\hline $\begin{array}{l}\text { Source of } \\
\text { Variation }\end{array}$ & Sum of Squares & df & Mean Square & $F$ \\
\hline Trials & 11.3 & 2 & 5.6 & 1.217 \\
Subjects & 368.6 & 19 & 19.4 & $4.217^{*}$ \\
S's Xtrials & 173.8 & $\frac{38}{59}$ & 4.6 & \\
\cline { 2 - 2 } & 553.7 & 59 & \\
\hline
\end{tabular}

* $p<.05$.

** $\mathrm{p}<.01$. 
Table 11

Analysis of Variance for the Well-being Scale

\begin{tabular}{lcrrr}
\hline $\begin{array}{l}\text { Source of } \\
\text { Variation }\end{array}$ & Sum of Squares & df & Mean Square & $f$ \\
\hline Trials & 130.6 & 2 & 65.3 & 4.324 \\
Subjects & 2830.9 & 19 & 148.9 & $9.861 * *$ \\
S's X trials & $\frac{572.5}{3}$ & $\frac{38}{59}$ & 15.1 & \\
$\quad$ Total & 3534.0 & & & \\
\hline
\end{tabular}

Table 12

Analysis of Variance for the Responsibility Scale

\begin{tabular}{lrrrr}
\hline $\begin{array}{l}\text { Source of } \\
\text { Variation }\end{array}$ & Sum of Squares & df & Mean Square & $F$ \\
\hline Trials & 52.9 & 2 & 26.4 & 2.778 \\
Subjects & 1456.5 & 19 & 76.6 & $8.063^{* *}$ \\
S's X trials & 360.3 & 38 & 9.5 & \\
\cline { 2 - 3 } & 1869.7 & 59 & & \\
\hline
\end{tabular}

Table 13

Analysis of Variance for the Socialization Scale

\begin{tabular}{lrrrr}
\hline $\begin{array}{l}\text { Source of } \\
\text { Variation }\end{array}$ & Sum of Squares & df & Mean Square & $F$ \\
\hline Trials & 62.7 & 2 & 31.3 & 1.490 \\
Subjects & 1022.3 & 19 & 53.8 & $2.561^{*}$ \\
S's X trials & $\frac{798.7}{1883.7}$ & $\frac{38}{59}$ & 21.0 & \\
Total & 188 & & \\
\hline
\end{tabular}

* $\quad \mathrm{p}<.05$ 
Table 14

Analysis of Variance for the Self-control Scale

\begin{tabular}{lcrrr}
\hline $\begin{array}{l}\text { Source of } \\
\text { Variation }\end{array}$ & Sum of Squares & df & Mean Square & $F$ \\
\hline Trials & 20.4 & 2 & 10.2 & .029 \\
Subjects & 2150.0 & 19 & 113.1 & $3.316^{* *}$ \\
Sis X trials & $\frac{1296.6}{3467.0}$ & $\frac{38}{59}$ & 34.1 & \\
$\quad$ Total & 346 & & \\
\hline
\end{tabular}

Table 15

Analysis of Variance for the Tolerance Scale

\begin{tabular}{lcccc}
\hline $\begin{array}{l}\text { Source of } \\
\text { Variation }\end{array}$ & Sum of Squares & df & Mean Square & $F$ \\
\hline Trials & 50.6 & 2 & 25.3 & 1.888 \\
Subjects & 1363.2 & 19 & 71.7 & $5.351^{*}$ \\
S's X trials & 509.8 & $\frac{38}{59}$ & 13.4 & \\
$\quad$ Total & 1923.6 & 59 & & \\
\hline
\end{tabular}

Table 16

Analysis of Variance for the Good Impression Scale

\begin{tabular}{lcrrr}
\hline $\begin{array}{l}\text { Source of } \\
\text { Variation }\end{array}$ & Sum of Squares & df & Mean Square & $F$ \\
\hline Trials & 10.3 & 2 & 5.1 & .190 \\
Subjects & 1414.1 & 19 & 74.4 & $2.776^{* *}$ \\
S's X trials & 1018.5 & $\frac{38}{59}$ & 26.8 & \\
$\quad$\begin{tabular}{l} 
Total \\
\hline
\end{tabular} & 2442.9 & & & \\
\hline
\end{tabular}

* $p<.05$.

** $\mathrm{p}<.01$. 
Table 17

Analysis of Variance for the Communality Scale

\begin{tabular}{lcrrr}
\hline $\begin{array}{l}\text { Source of } \\
\text { Variation }\end{array}$ & Sum of Squares & df & Mean Square & $F$ \\
\hline Trials & 28.2 & 2 & 14.1 & 1.516 \\
Subjects & 1147.9 & 19 & 60.4 & $6.494^{*}$ \\
S's X trials & 354.9 & $\frac{38}{59}$ & 9.3 & \\
$\quad$ Total & 1531.0 & & & \\
\hline
\end{tabular}

Table 18

Analysis of Variance for the Achievement via Conformity Scale

\begin{tabular}{lcrrr}
\hline $\begin{array}{l}\text { Source of } \\
\text { Variation }\end{array}$ & Sum of Squares & df & Mean Square & $F$ \\
\hline Trials & 6.6 & 2 & 3.3 & .221 \\
Subjects & 1080.4 & 19 & 56.9 & $3.818^{* *}$ \\
S's X trials & $\frac{566.6}{1653.6}$ & $\frac{38}{59}$ & 14.9 & \\
$\quad$ Total & 163.6 & & \\
\hline
\end{tabular}

Table 19

Analysis of Variance for the Achievement via Independence Scale

\begin{tabular}{lcrrr}
\hline $\begin{array}{l}\text { Source of } \\
\text { Variation }\end{array}$ & Sum of Squares & df & Mean Square & $F$ \\
\hline Trials & 21.6 & 2 & 10.8 & 3.495 \\
Subjects & 861.9 & 19 & 45.4 & $11.641^{*}$ \\
S's X trials & 149.5 & 38 & 3.9 & \\
\cline { 2 - 4 } & 1033.0 & 59 & & \\
\hline
\end{tabular}

* $p<.05$.

$* * p<.01$. 
Table 20

Analysis of Variance for the Intellectual Efficiency Scale

\begin{tabular}{lcrrrr}
\hline $\begin{array}{l}\text { Source of } \\
\text { Variation }\end{array}$ & Sum of Squares & df & Mean Square & $F$ \\
\hline Trials & 98.4 & 2 & 49.2 & 4.032 \\
Subjects & 2103.3 & 19 & 110.7 & $9.074^{* *}$ \\
S's Xtrials & $\frac{465.3}{2667.0}$ & $\frac{38}{59}$ & 12.2 & \\
$\quad$ Total & 59 & \\
\hline
\end{tabular}

Table 21

Analysis of Variance for the Psychological-mindedness Scale

\begin{tabular}{lrrrr}
$\begin{array}{l}\text { Source of } \\
\text { Variation }\end{array}$ & Sum of Squares & df & Mean Square & $F$ \\
\hline Trials & 9.1 & 2 & 4.5 & 2.812 \\
Subjects & 538.2 & 19 & 28.3 & $17.687^{* *}$ \\
S's X trials & 60.0 & $\frac{38}{59}$ & 1.6 & \\
Total & 607.3 & & & \\
\hline
\end{tabular}

Table 22

Analysis of Variance for the Flexibility Scale

\begin{tabular}{lcrrr}
\hline $\begin{array}{l}\text { Source of } \\
\text { Variation }\end{array}$ & Sum of Squares & df & Mean Square & $F$ \\
\hline Trials & 13.5 & 2 & 6.7 & .893 \\
Subjects & 549.1 & 19 & 28.9 & $3.853^{* *}$ \\
S's X trials & $\frac{284.3}{846.9}$ & $\frac{38}{59}$ & 7.5 & \\
Total & 8 & & \\
\hline
\end{tabular}

* $p<.05$.

** $p<.01$. 
Table 23

Analysis of Variance for the Femininity Scale

\begin{tabular}{lcrrr}
\hline $\begin{array}{l}\text { Source of } \\
\text { Variation }\end{array}$ & Sum of Squares & df & Mean Square & $f$ \\
\hline Trials & 10.9 & 2 & 5.4 & 1.227 \\
Subjects & 334.1 & 19 & 17.6 & $4.000^{* *}$ \\
S's X trials & $\frac{166.9}{511.9}$ & $\frac{38}{59}$ & 4.4 & \\
$\quad$ Total & 511.9 & & \\
\hline
\end{tabular}

Tables 24 through 35 show the trend analyses for the Jesness Inventory. Again, trend significance is not reached; and subject variability is significant on all but two scales and the Asocial Index (A/I).

Table 24

Analysis of Variance for the Social Maladjustment Scale (Weighted Items)

\begin{tabular}{lrrrr}
\hline $\begin{array}{l}\text { Source of } \\
\text { Variation }\end{array}$ & Sum of Squares & df & Mean Square & F \\
\hline Trials & 54.8 & 2 & 27.4 & 1.971 \\
Subjects & 1437.4 & 19 & 75.6 & $5.439^{* *}$ \\
S's X trials & 528.4 & $\frac{38}{59}$ & 13.9 & \\
\cline { 2 - 3 } Total & 2020.6 & 59 & \\
\hline
\end{tabular}

* $\mathrm{p}<.05$.

** $p<.01$. 
Table 25

Analysis of Variance for the Social Maladjustment Scale

\begin{tabular}{lcrcc}
\hline $\begin{array}{l}\text { Source of } \\
\text { Variation }\end{array}$ & Sum of Squares & df & Mean Square & $F$ \\
\hline Trials & 38.5 & 2 & 19.2 & .927 \\
Subjects & 1852.9 & 19 & 97.5 & $4.710^{* *}$ \\
S's X trials & $\frac{785.9}{2677.3}$ & $\frac{38}{59}$ & 20.7 & \\
$\quad$ Total & 2677.3 & & & \\
\hline
\end{tabular}

Table 26

Analysis of Variance for the Value Orientation Scale

\begin{tabular}{lcrrr}
\hline $\begin{array}{l}\text { Source of } \\
\text { Variation }\end{array}$ & Sum of Squares & df & Mean Square & $F$ \\
\hline Trials & 13.0 & 2 & 6.5 & .293 \\
Subjects & 3033.2 & 19 & 159.6 & $7.189 * *$ \\
S's X trials & $\frac{842.8}{3889.0}$ & $\frac{38}{59}$ & 22.2 & \\
$\quad$ Total & 389.0 & & \\
\hline
\end{tabular}

Table 27

Analysis of Variance for the Immaturity Scale

\begin{tabular}{lcccc}
\hline $\begin{array}{l}\text { Source of } \\
\text { Variation }\end{array}$ & Sum of Squares & df & Mean Square & $F$ \\
\hline Trials & 1.6 & 2 & .8 & .06 \\
Subjects & 514.2 & 19 & 27.1 & 2.037 \\
S's X trials & $\underline{506.8}$ & $\frac{38}{59}$ & 13.3 & \\
$\quad$ Total & 1022.6 & 59 & & \\
\hline
\end{tabular}

$* P<.05$.

$* * p<.01$. 
Table 28

Analysis of Variance for the Autism Scale

\begin{tabular}{lrrrrr}
\hline $\begin{array}{l}\text { Source of } \\
\text { Variation }\end{array}$ & Sum of Squares & df & Mean Square $F$ \\
\hline Trials & 28.8 & 2 & 14.4 & 2.117 \\
Subjects & 579.9 & 19 & 30.5 & $4.485^{* *}$ \\
S's X trials & $\frac{260.3}{869.0}$ & $\frac{38}{59}$ & 6.8 & \\
$\quad$ Total & 869.6 & \\
\hline
\end{tabular}

Table 29

Analysis of Variance for the Alienation Scale

\begin{tabular}{lrrrr}
\hline $\begin{array}{l}\text { Source of } \\
\text { Variation }\end{array}$ & Sum of Squares & df & Mean Square & $f$ \\
\hline Trials & .9 & 2 & .4 & .048 \\
Subjects & 905.8 & 19 & 47.7 & $5.678^{* *}$ \\
S's Xtrials & $\frac{318.2}{224.9}$ & $\frac{38}{59}$ & 8.4 & \\
Total & 122.9 & & \\
\hline
\end{tabular}

Table 30

Analysis of Variance for the Manifest Aggression Scale

\begin{tabular}{|c|c|c|c|c|}
\hline $\begin{array}{l}\text { Source of } \\
\text { Variation }\end{array}$ & Sum of Squares & $d f$ & Mean Square & $\mathbf{F}$ \\
\hline Trials & 22.3 & 2 & 11.1 & .745 \\
\hline Subjects & 1850.8 & 19 & 97.4 & $6.536 * *$ \\
\hline s's X trials & 566.2 & 38 & 14.9 & \\
\hline Total & 2439.3 & 59 & & \\
\hline
\end{tabular}


Table 31

Analysis of Variance for the Withdrawal Scale

\begin{tabular}{lcrrr}
\hline $\begin{array}{l}\text { Source of } \\
\text { Variation }\end{array}$ & Sum of Squares & df & Mean Square $F$ \\
\hline Trials & 2.7 & 2 & 1.3 & .213 \\
Subjects & 399.9 & 19 & 21.0 & $3.443^{*}$ \\
S's X trials & $\frac{232.3}{634.9}$ & $\frac{38}{59}$ & 6.1 & \\
Total & 634 & & \\
\hline
\end{tabular}

Table 32

Analysis of Variance for the Social Anxiety Scale

\begin{tabular}{lcrrr}
\hline $\begin{array}{l}\text { Source of } \\
\text { Variation }\end{array}$ & Sum of Squares & df & Mean Square & $f$ \\
\hline Trials & 11.5 & 2 & 5.7 & .475 \\
Subjects & 666.1 & 19 & 35.0 & $2.916^{* *}$ \\
S's X trials & $\underline{456.3}$ & $\underline{38}$ & 12.0 & \\
\multicolumn{1}{r}{ Total } & 1127.9 & 59 & & \\
\hline
\end{tabular}

Table 33

Analysis of Variance for the Repression Scale

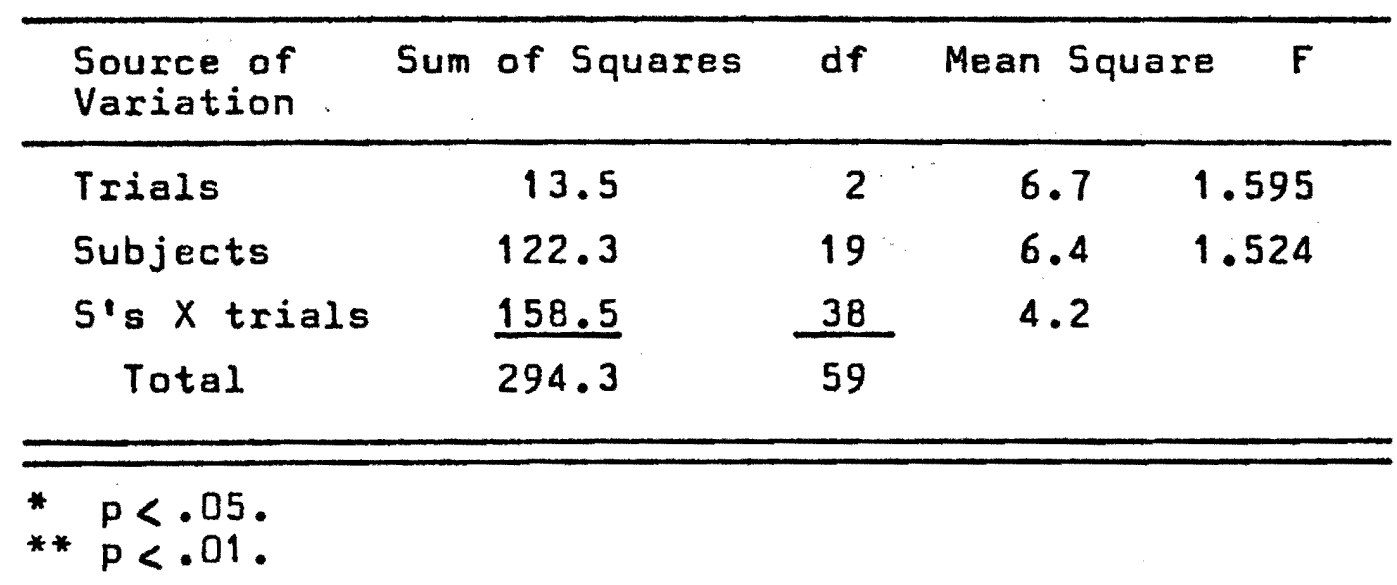


Table 34

Analysis of Variance for the Denial Scale

\begin{tabular}{lcrrr}
\hline $\begin{array}{l}\text { Source of } \\
\text { Variation }\end{array}$ & Sum of Squares & df & Mean Square $F$ \\
\hline Trials & 3.5 & 2 & 1.7 & .144 \\
Subjects & 825.0 & 19 & 43.4 & $3.678^{* *}$ \\
S's Xtrials & 450.2 & $\frac{38}{11.8}$ & 11 \\
Total & 1278.7 & 59 & \\
\hline
\end{tabular}

Table 35

Analysis of Variance for the Asocial Index

\begin{tabular}{|c|c|c|c|c|}
\hline $\begin{array}{l}\text { Source of } \\
\text { Variation }\end{array}$ & Sum of Squares & $d f$ & Mean Square & $\mathbf{F}$ \\
\hline Trials & 20.0 & 2 & 10.0 & .510 \\
\hline Subjects & 398.3 & 19 & 21.0 & 1.071 \\
\hline s's X trials & 743.9 & 38 & 19.6 & \\
\hline Total & 1162.2 & 59 & & \\
\hline
\end{tabular}

The ratio of variances between the first, second and last tests on the CPI are shown in Table 36. None of these ratios is significant. Table 37 shows the ratio of variances over the three test administrations on the Jesness Inventory. A significant difference in the variability of change occurs on the Asocial Index, indicating that some girls are becoming more delinquency prone while some girls are becoming less so. 
Table 36

Variance Ratios on the CPI over Three Administrations

\begin{tabular}{|c|c|c|c|c|c|c|}
\hline Scale & $\begin{array}{c}\text { First } \\
\text { Test }\end{array}$ & $\begin{array}{l}\text { Second } \\
\text { Test }\end{array}$ & $\mathbf{F}$ & $\begin{array}{c}\text { First } \\
\text { Test }\end{array}$ & $\begin{array}{c}\text { Third } \\
\text { Test }\end{array}$ & $\mathbf{F}$ \\
\hline $\begin{array}{l}\text { Do } \\
\text { Cs } \\
5 y \\
5 p \\
\text { Sa } \\
\text { Wb }\end{array}$ & $\begin{array}{r}43.0 \\
14.8 \\
24.7 \\
13.9 \\
8.4 \\
64.2\end{array}$ & $\begin{array}{l}42.8 \\
20.3 \\
36.7 \\
32.2 \\
10.0 \\
63.1\end{array}$ & $\begin{array}{l}---37 \\
1.48 \\
2.32 \\
1.19 \\
-\ldots-\end{array}$ & $\begin{array}{r}43.0 \\
14.8 \\
24.7 \\
13.9 \\
8.4 \\
64.2\end{array}$ & $\begin{array}{l}43.5 \\
21.7 \\
30.0 \\
26.2 \\
10.1 \\
51.8\end{array}$ & $\begin{array}{l}1.01 \\
1.47 \\
1.21 \\
1.88 \\
1.2 \\
-. .2\end{array}$ \\
\hline $\begin{array}{l}\text { Re } \\
\text { So } \\
\text { Sc } \\
\text { To } \\
\text { Gi } \\
\text { Cm }\end{array}$ & $\begin{array}{l}28.5 \\
35.3 \\
64.2 \\
28.1 \\
36.1 \\
31.0\end{array}$ & $\begin{array}{l}36.7 \\
22.9 \\
49.4 \\
35.1 \\
41.8 \\
25.3\end{array}$ & $\begin{array}{l}1.29 \\
-.0- \\
0 .-2 \\
1.25 \\
1.16 \\
\ldots-.\end{array}$ & $\begin{array}{l}28.5 \\
35.3 \\
64.2 \\
28.1 \\
36.1 \\
31.0\end{array}$ & $\begin{array}{l}30.4 \\
38.7 \\
68.3 \\
35.3 \\
50.1 \\
22.7\end{array}$ & $\begin{array}{l}1.07 \\
1.1 \\
1.06 \\
1.26 \\
1.39 \\
\ldots--\end{array}$ \\
\hline $\begin{array}{l}A c \\
A i \\
I e \\
P_{y} \\
F x \\
F_{e}\end{array}$ & $\begin{array}{r}21.2 \\
16.4 \\
35.8 \\
7.4 \\
12.0 \\
9.6\end{array}$ & $\begin{array}{r}20.5 \\
19.7 \\
39.6 \\
9.0 \\
13.3 \\
11.5\end{array}$ & $\begin{array}{l}---- \\
1.2 \\
1.11 \\
1.22 \\
1.11 \\
1.2\end{array}$ & $\begin{array}{r}21.2 \\
16.4 \\
35.8 \\
7.4 \\
12.0 \\
9.6\end{array}$ & $\begin{array}{r}45.0 \\
17.0 \\
59.7 \\
15.1 \\
18.4 \\
5.3\end{array}$ & $\begin{array}{l}2.12 \\
1.04 \\
1.67 \\
2.04 \\
1.53 \\
-.-2\end{array}$ \\
\hline
\end{tabular}

Table 37

Variance Ratios on the Jesness Inventory

\begin{tabular}{|c|c|c|c|c|c|c|}
\hline Scale & $\begin{array}{c}\text { First } \\
\text { Test }\end{array}$ & $\begin{array}{c}\text { Second } \\
\text { Test }\end{array}$ & $F$ & $\begin{array}{c}\text { First } \\
\text { Test }\end{array}$ & $\begin{array}{c}\text { Third } \\
\text { Test }\end{array}$ & $\mathbf{F}$ \\
\hline $\begin{array}{l}S M x \\
S M \\
V D \\
I \mathrm{~mm} \\
\text { Au } \\
\text { AI }\end{array}$ & $\begin{array}{l}30.2 \\
35.0 \\
64.5 \\
16.0 \\
17.8 \\
15.8\end{array}$ & $\begin{array}{l}29.7 \\
37.9 \\
62.9 \\
23.0 \\
15.7 \\
28.4\end{array}$ & $\frac{1.08}{1.44}$ & $\begin{array}{l}30.2 \\
35.0 \\
64.5 \\
16.0 \\
17.8 \\
15.8\end{array}$ & $\begin{array}{l}43.5 \\
65.9 \\
76.6 \\
14.7 \\
10.7 \\
20.3\end{array}$ & $\begin{array}{l}1.44 \\
1.88 \\
1.19 \\
-1 .-0 \\
1.28\end{array}$ \\
\hline $\begin{array}{l}\text { MA } \\
\text { Wd } \\
\text { SA } \\
\text { Rep } \\
\text { Den } \\
A / I\end{array}$ & $\begin{array}{r}47.3 \\
10.9 \\
20.4 \\
5.2 \\
22.0 \\
10.4\end{array}$ & $\begin{array}{r}33.1 \\
8.3 \\
9.1 \\
5.8 \\
18.9 \\
17.9\end{array}$ & $\begin{array}{l}--- \\
---0 \\
-1.11 \\
-1.72\end{array}$ & $\begin{array}{r}47.3 \\
10.9 \\
20.4 \\
5.2 \\
22.0 \\
10.4\end{array}$ & $\begin{array}{r}46.8 \\
14.1 \\
29.2 \\
3.7 \\
26.1 \\
31.8\end{array}$ & $\begin{array}{l}1.29 \\
1.43 \\
-1.19 \\
3.06\end{array}$ \\
\hline
\end{tabular}

* $\mathrm{p}<.05$.

** $\mathrm{p}<.01$. 
Figure 1 shows the sample divided into high, middle and low scorers on the Asocial Index $(A / I)$ at the time of the initial administration. At the third month interval, the high scoring group scores lower than the other two groups. The middle group mean remains somewhat the same, while the low scorers show an appreciable increase. At the six-month stage, the high and middle groups show a regression toward the mean. The low scorers return to low-score position (least delinquent), but not completely to their original mean. The observed improvement in high scorers was found to be highly significant $(t=5.23,12 \mathrm{df})$.

\section{Figure 1}

High, Middle and Low Scorers on the Asocial Index over Six Months

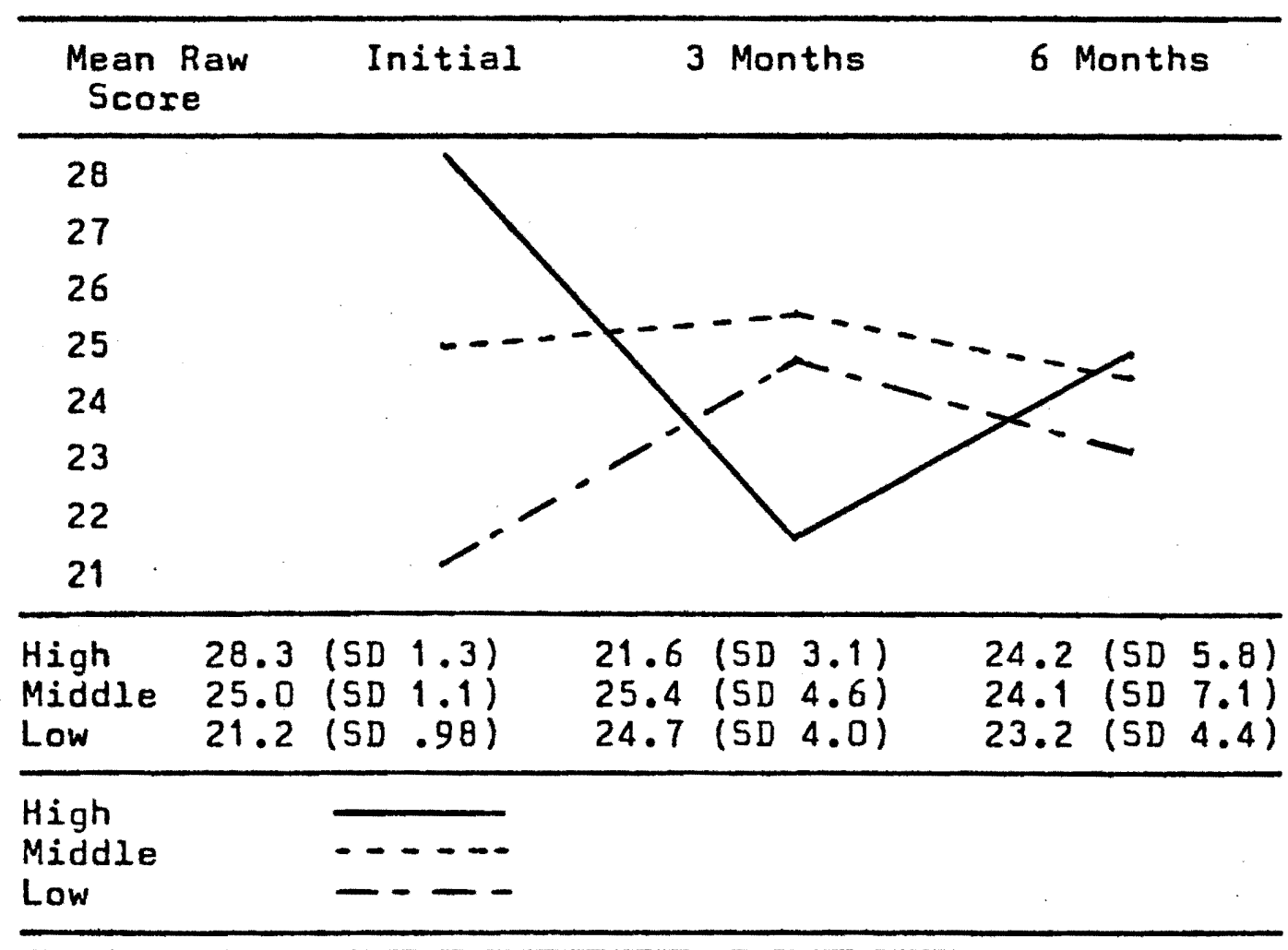


Figures 2,3 and 4 show the increases and decreases on the Asocial Index for each subject in each of the three sub groups between initial and final scores.

\section{Figure 2}

Changes in Delinquency Proneness in High Scorers

\begin{tabular}{lcccc}
\hline Subject & $\begin{array}{c}\text { Initial } \\
\text { Score }\end{array}$ & $\begin{array}{c}\text { Final } \\
\text { Score }\end{array}$ & Increase & Decrease \\
\hline 101 & 28 & 21 & 9 & 7 \\
102 & 27 & 36 & & 7 \\
103 & 27 & 20 & 20 & 10 \\
104 & 30 & 27 & 3 \\
105 & 30 & 21 & 7 \\
106 & 28 & 25 & 3 \\
107 & 28 & 25 & \\
\hline$N=7$ &
\end{tabular}

Figure 3

Changes in Delinquency Proneness in Middle Scorers

\begin{tabular}{lcccc}
\hline Subject & $\begin{array}{c}\text { Initial } \\
\text { Score }\end{array}$ & $\begin{array}{c}\text { Final } \\
\text { Score }\end{array}$ & Increase & Decrease \\
\hline 108 & 26 & 16 & & 10 \\
109 & 24 & 13 & & 11 \\
110 & 26 & 27 & 1 & \\
111 & 25 & 33 & 8 & \\
112 & 23 & 28 & 5 & - \\
113 & 26 & 27 & 1 & \\
114 & 25 & 25 & - & \\
\hline$N=7$ & & & & \\
\hline
\end{tabular}


Figure 4

Changes in Delinquency Proneness in Low Scorers

\begin{tabular}{lcccc}
\hline Subject & $\begin{array}{c}\text { Initial } \\
\text { Score }\end{array}$ & $\begin{array}{c}\text { Final } \\
\text { Score }\end{array}$ & Increase & Decrease \\
\hline 115 & 20 & 27 & 7 & \\
116 & 22 & 28 & 6 & \\
117 & 22 & 21 & & 4 \\
118 & 20 & 16 & & 4 \\
119 & 21 & 23 & 2 & \\
120 & 22 & 24 & 2 & \\
\hline$N=6$ & & \\
\hline
\end{tabular}


CONCLUSIONS

In comparing the Maple Lane girls to Gough's high school norm group, the subjects differed in areas descriptive of delinquent behavior; such as inability and/or unwillingness to conform ( $\mathrm{Cm}, A c)$, low self-control (Sc), lack of responsibility (Re), aggressiveness (Fe), disregard for social conventions and rules (5o, $\mathrm{Cm}$ ), and a. lack of concern over the reactions of others (Gi).

The lower Intellectual Efficiency Scale scores are not simply an indication of lower intelligence: they reflect the disrupted schooling typical of delinquent careers and relate to the depressed sense of well-being (Wb) ane might experience after a number of court appearances, removal from home and periods of detention, followed by commitment to a state institution.

Dominance (Do), sociability $(S y)$, social poise (Sp), ability to achieve independently ( $A$ i), sensitivity to the needs of others $(P y)$ and flexibility $(F x)$ do not appear to differentiate between delinquents and nondelinquents. Another way of looking at this is to say Maple Lane girls are not without social and interpersonal skills. On the other hand, they are not just like other high school students, who simply happen to have been caught doing wrong.

The significant gain in self-acceptance (5a) cannot be explained without clinical interpretation of 
the individual profiles. Whether this is a matter of treatment gains or an increase in ego-syntonic defense systems will have to remain an undecided question in this study. The question is further confounded by insufficient validation of the scale (Megargee, 1972). One of the School's goals is to help its residents become like their normal counterparts; that is, less delinquent and more capable of adequate functioning. The findings suggest that the impact of being in the institution for six months results in small, but statistically insignificant gains. Repeating the study with a larger sample would establish whether these trends are real or not.

"One of the few agreed-upon 'facts' in the field of corrections is that offenders are not all alike. That is, they differ from each other, not only in the form of their offense, but also in the reasons for and the meaning of their crime." (Warren, 1969)

It was no surprise therefore, to find that the Maple Lane girls also differ widely in personality traits and degree of delinquent identification. That this subject variability was not found on the Immaturity (Imm) and Repression (Rep) Scales and on the Asocial Index $(A / I)$ seems due to random variation, rather than to any meaningful variable effect.

The expected variability of change at the end of six months is substantiated on only one measure. This lack of increased variability seems to be due to the small sample size, rather than to six months being too 
short an interval of time. The significant variability on the Asocial Index is not considered to be simply a matter of chance. The outstanding feature of the Jesness Inventory is the Asocial Index, which was designed to predict delinquency and to precisely reflect changes in delinquent attitudes and identification over short periods of time.

The fact that high scorers (the most delinquent) showed highly significant improvement, while low scorers may have become more delinquent by the end of three months suggests that the Maple Lane program is most effective with severe delinquents; while it may be harmful for girls who are only marginally delinquent. This in part upholds Bergin's concept of a deterioration effect. and is an area of concern which should be further examined.

If this study were to be repeated, the sample size should be increased. Patterns and rates of change vary with the individual girls and are only partially reflected in this study because of its limitation to six months. Larger sample size is particularly crucial for comparing the demographic variables such as age, race, cottage placement and priox institutional experience between those girls who seem to be improving and those showing deterioration. 
This report does not reflect the numerous questions pertaining to program considerations at Maple Lane that can be raised by a closer examination of the data. Some girls showed gains by scoring as much as two standard deviations higher in certain areas on the CPI while their delinquency proneness remained largely unmodified. Other girls were seemingly being rewarded for improvement by the granting of furloughs, while their test scores did not reveal a pattern of improvement. Questions of when, and which attitudes are being reinforced are not answered by this study, but it does provide enough information to raise this as an issue to be resolved.

To examine the correlations between scores on the two inventories and how they relate to reports on behavior was not one of the purposes of this project. It does remain, however, an area that may yield useful information to the staff as they try to determine whether or not their program provides for gains in those areas they feel are most related to a girl living successfully in the community.

How does one develop accompanying attitudes that alters "psychological mindedness" from a potentially expoitative skill to a strength that will eventually enhance impulse control? If the residents' sense of well-being could be raised, would this have a positive or negative effect in their overall functioning? How is it that the girls are more inclined to "fake bad" 
than to "fake good"? Are the girls who are showing the greatest improvement getting some kind of support from their families that the others are not?

What is there in the experience of regularly. attending the academic school that results in some girls making tremendous gains in "intellectual efficiency", while others function even less well than when they were admitted? Is it an experience for the latter that only reinforces past failures? How are relationships with staff and peers effected by a girl's "social presence" and "sociability"? Will a girl who is low in these areas find the same opportunities at the School as a girl who is high is these skills?

These are just a few of the issues that enter into treatment considerations and policy decisions. Certainly, they must be examined in terms of setting goals and evaluating the effectiveness of the program at Maple Lane, or in any comparable endeavor. 


\section{SELECTED BIBLIOGRAPHY}

Bergin, Allen E. "The Effects of Psychotherapy: Negative Results Revisited." Journal of Counseling Psychology, Vol. 10, No. 3 $(1963), 244-250$.

Bergin, Allen E. "Some Implications of Psychotherapy Research for Therapeutic Practice." Journal of Abnormal Psychology, Vol. 71 , No. 4 (August 1966), 235-246.

Bergin, Allen E. "The Deterioration Effect: A Reply to Braucht." Journal of Abnormal Psychology. Vol. 75, No. 3 (June 1970), 300-302.

Campos, Leonard P. "Developing Eight Therapeutic Communities' at a School for Boys." California Youth Authority Quarterly, Vol. 20, No. 2 (1967), 20-31.

Canter, Francis M. "Simulation on the California Psychological Inventory and the Adjustment of the Simulator." Journal of Consulting Psychology, Vol. 27, No. 3 (June 1963), 253-256.

Edwards, Allen L. Experimental Design in Psychological Research. revised edition. New York: Holt, Rinehart and Winston, 1960.

Edwards, Allen L. Statistical Methods for the Behavioral Sciences. New York: Rinehart \& Company, Inc., 1954.

Gough, Harrison G. "A Cross-cultural Analysis of the CPI Femininity Scale." Consulting Psychology, Vol. 30, No. 2 (April 1966), 136-141.

Gough, Harrison G. Manual for the California Psychological Inventory. revised 1964, 1969. Palo Alto, California: Consulting Psychologists Press, Inc., 1957. 
Gough, Harrison G. "Scoring High on an Index of Social Maturity." Journal of Abnormal Psychology. Vol. 77, No. 3 (June 1971), 236-241.

Guttman, Evelyn S. MMPI-Measured Changes in Treated and Untreated Youth Authority Wards Judged in Need of Psychiatric Treatment. Research Report No. 25. Publication of the California Youth Authority, Division of Research, November 1961.

Haan, Norma. "Coping and Defense Mechanisms Related to Personality Inventories." Journal of Consulting Psychology. Vol. 29, No. 4 (August 1965), 373-378.

Jesness, Carl F. Manual: The Jesness Inventory. Palo Alto, California: Consulting Psychologists Press, 1966.

Jesness, Carl F. The Jesness Inventory: Development and Validation. Research Report No. 29. Publication of the California Youth Authority, Division of Research, January 1962.

Jesness, Carl F. Redevelopment and Revalidation of the Jesness Inventory. Research Report No. 35. Publication of the California Youth Authority, Division of Research, November 1963.

Jesness, Carl F. The Fricot Ranch Study: Outcomes with Small versus Large Living Groups in the Rehabilitation of Delinquents. Research Report No. 47. Publication of the California Youth Authority, Division of Research, October 1965.

Jesness, Carl F. "The Preston Typology Study." California Youth Authority Quarterly. Vol. 23, No. 4 (1970), 26-38.

Lindquist, E.F. Design and Analysis of Experiments in Psychology and Education. Boston: Houghton Mifflin Company, 1953.

Megargee, Edwin Inglee. The California Psychological Inventory Handbook. San Francisco. Washington. London: Jossey-Bass Inc., Publishers, 1972. 
Rose, Gordon. "Early Identification of Delinquents." British Journal of Criminolagy. Vol. 7 No. $1(1967), 6-35$.

Seckel, Joachim P. Experiment in Group Counseling at

Two Youth Authority Institutions. Research

Report No. 46. Publication of the

California Youth Authority, Division of

Research, September 1965.

Sowles, Richard C. and Gill, John H. "Institutional and Community Adjustment of Delinquents

Following Counseling." Journal of

Consulting and Clinical Psychology.

Vol. 34, No. 3 (June 1970), 398-402.

State of Washington. Statistical Report for Office of

Juvenile Rehabilitation: Fiscal Year 1970.

Department of Social and Health Services,

Division of Institutions, Dffice of

Research.

Warren, Marguerite Q. (principal investigator).

Community Treatment Project: Comparative

Assessment of Institutional Treatment for

Comparable Groups of Youth Authority Wards.

Research Report No. 7. Publication of the

California Youth Authority, Division of

Research, 1966.

Warren, Marguerite $Q$. "The Case for Differential

Treatment of Delinquents." Proceedings

of the Ninth Annual Research Meeting.

State of Washington: Department of

Institutions, Division of Research,

April 1969. 
Appendix 1

California Psychological Inventory Scales: Brief Definition and Purpose

Do (dominance) - 46 items. To assess factors of leadership ability, dominance, persistence and social initiative.

Cs (capacity for status) - 32 items. To serve as an index of an individual's capacity for status (not his actual or achieved status). The scale attempts to measure the personal qualities and attributes which underlie and lead to status.

Sy (sociability) - 36 items. To identify persons of outgoing, sociable, participative temperament.

Sp (social presence) - 56 items. To assess factors such as poise, spontaneity, and self-confidence in personal and social interaction.

Sa (self-acceptance) - 34 items. To assess factors such as sense of personal worth, self-acceptance, and capacity for independent thinking and action.

Wb (sense of well-being) - 44 items. To identify persons who minimize their worries and complaints, and who are relatively free from self-doubt and disillusionment.

Re (responsibility) - 42 items. To identify persons of conscientious, responsible, and dependable disposition and temperament.

So (socialization) -- 54 items. To indicate the degree of social maturity, integrity, and rectitude which the individual has attained.

Sc (self-control) - 50 items. To assess the degree and adequacy of self-regulation and self-control and freedom from impulsivity and self-centeredness.

To (tolerance) -- 32 items. To identify persons with permissive, accepting, and non-judgmental social beliefs and attitudes.

Gi (good impression) -- 40 items. To identify persons capable of creating a favorable impression, and who are concerned about how others react to them. 
Cm (communality) - 28 items. To indicate the degree to which an individual's reactions and responses correspond to the modal ("common") pattern established for the inventory.

Ac (achievement via conformance) -- 38 items. To identify those factors of interest and motivation which facilitate achievement in any setting where conformance is a positive behavior.

Ai (achievement via independence) -- 32 items. To identify those factors of interest and motivation which facilitate achievement in any setting where autonomy and independence are positive behaviors.

Ie (intellectual efficiency) - 52 items. To indicate the degree of personal and intellectual efficiency which the individual has attained.

Py (psychological-mindedness) -- 22 items. To measure the degree to which the individual is interested in, and responsive to, the inner needs, motives, and experiences of others.

Fx (flexibility) -- 22 items. To indicate the degree of flexibility and adaptability of a person's thinking and social behavior.

Fe (femininity) -- 38 items. To assess the masculinity or femininity of interests. (High scores indicate more feminine interests, low scores more masculine.)

\footnotetext{
* Quoted from the Manual for the California Psychological Inventory; by Harrison G. Gough, Ph.D., 1957. Revised 1964, 1969.
} 
Appendix 2

The Jesness Inventory: Brief Definition of the Scales

Social Maladjustment Scale (SM) -- 63 items. Social Maladjustment refers here to a set of attitudes associated with inadequate or disturbed socialization, as defined by the extent to which an individual shares the attitudes of persons who demonstrate inability to meet environmental demands in socially approved ways. Several items (31) which showed unusual discriminating power between the delinquent and non-delinquent groups were given added weight in computing the Asocialization score. The weighted items are designated SMx.

Value Orientation Scale (VO) -- 39 items. Value Orientation refers to a tendency to share attitudes and opinions characteristic of persons in the lower socioeconomic classes.

Immaturity Scale ( $\mathrm{Imm}$ ) - 45 items. Immaturity reflects the tendency to display attitudes and perceptions of self and others which are usual for persons of a younger age than the subject.

Autism Scale (Au) - 28 items. Autism measures a tendency, in thinking and perceiving, to distort reality according to one's personal desires or needs.

Alienation Scale (Al) - 26 items. Alienation refers to the presence of distrust and estrangement in a person's attitudes toward others, especially toward those representing authority.

Manifest Aggression (MA) - 31 items. Manifest Aggression reflects an awareness of unpleasant feelings, especially of anger and frustration, a tendency to react readily with emotion, and perceived discomfort concerning the presence and control of these feelings.

Withdrawal scale (Wd) - 24 items. Withdrawal involves a perceived lack of satisfaction with self and others and a tendency toward isolation from others.

Social Anxiety Scale (SA) - 24 items. Social Anxiety refers to perceived emotional discomfort associated with interpersonal relationships. 
Repression Scale (Rep) -- 15 items. Repression reflects the exclusion from conscious awareness of feelings and emotions which the individual normally would be expected to experience, or his failure to label these emotions.

Denial Scale (Den) -- 20 items. Denial indicates a reluctance to acknowledge unpleasant events or aspects of reality often encountered in daily living.

Asocial Index. Asocialization refers to a generalized disposition to resolve problems of social and personal adjustment in ways ordinarily regarded as showing a disregard for social customs or rules.

* Quoted from the Manual for the Jesness Inventory, by Carl F. Jesness, Ph.D., 1966. 